Original Research Paper

\title{
In silico Assessment of the Arg85Trp Glucokinase Mutation Effects
}

\author{
${ }^{1,2}$ Faiza Bouldjennet, ${ }^{1}$ Souhila Bouaziz-Terrachet, ${ }^{3}$ Malha Azzouz and ${ }^{2}$ Rachida Raache \\ ${ }^{I}$ Faculty of Sciences, University M'Hamed Bougara of Boumerdes, Algeria \\ ${ }^{2}$ Laboratory of Cellular and Molecular Biology, Faculty of Biological Sciences, \\ University of Sciences and Technology Houari Boumediene, Algiers, Algeria \\ ${ }^{3}$ Department of Diabetology, Mustapha Pacha Hospital, Algiers, Algeria
}

Article history

Received: 25-12-2019

Revised: 11-02-2020

Accepted: 24-02-2020

Corresponding Author:

Faiza Bouldjennet

Faculty of Sciences, University

M'Hamed Bougara of

Boumerdes, Algeria

Email: fai.bouldjennet@gmail.com

\begin{abstract}
Maturity Onset Diabetes of the Young (MODY) is a heterogeneous group of autosomal dominantly inherited, young-onset $\beta$ cell disorders that account for approximately $2 \%$ of non-insulindependent diabetics in Europe. MODY2 or GCK-MODY is caused by inactivating heterozygous mutations in the glucokinase. Linkage of MODY to the Arg85Trp GCK mutation, among Algerian diabetic patients has been established in our previous research. The main objective of this study is to strengthen the evidence for the pathogenicity of this mutation using bioinformatics tools. For this purpose, prediction of Arg85Trp mutation effect on glucokinase (PDB ID: 1V4T) stability was performed using I-Mutant 3.0, PoPMuSiC 3.1, DUET and mCSM web servers. Otherwise, structural analysis was performed after optimization of the native and the mutated structures (PDB ID: 1V4T). For that the steepest descent geometries optimization were applied using Nano Molecular Dynamics software (NAMD 2.12). Subsequently, interactions established between the native Arg85 or the mutated Trp85 and the surrounding residues were studied using Accelrys Discovery Studio Visualizer software. In silico analysis conducted through I-Mutant 3.0, PoPMuSiC 3.1, DUET and mCSM softwares, predicts the Arg85Trp as a destabilizing mutation. Structural modeling gives further evidence in favor of the pathogenicity of this mutation. Overall, our results corroborate with the previous metabolic and in silico studies which associate Arg85Trp mutation to MODY phenotype.
\end{abstract}

Keywords: In silico, Glucokinase, Arg85Trp Mutation, Stability, Structural Modeling

\section{Introduction}

Maturity Onset Diabetes of the Young (MODY) is a heterogeneous group of autosomal dominantly inherited, young-onset $\beta$-cell disorders that account for approximately $2 \%$ of non-insulin-dependent diabetics in Europe. Heterozygous inactivating mutations in the $G C K$ gene encoding the glucokinase have been linked to a subtype of Maturity Onset Diabetes of the Young (MODY) named MODY2 or GCK-MODY (OMIM. 125851) (Vionnet et al., 1992). GCK-MODY is the most common form of MODY diabetes in several countries, including Italy (Capuano et al., 2012; Massa et al., 2001), France (Chevre et al., 1998), Spain (Barrio et al., 2002; Estalella et al., 2007) and others.
In our previous investigation (Bouldjennet et al., 2019), we have demonstrated linkage of MODY to the Arg85Trp GCK mutation among Algerian diabetic patients. We have also revealed: (a) The high conservation of the arginine residue at position 85 of the glucokinase; (b) the probable effect of the Arg85Trp mutation on glucokinase structure/function and stability of the glucokinase (PDB: 1V4S) and (c) within the glucokinase, the substitution of the Arg85 by a Trp85 residue led to abolition/creation of interactions with the surrounding residues (Bouldjennet et al., 2019).

In this study we use bioinformatic approaches in order to strengthen the evidence for the pathogenicity of the detected variant. These approaches were performed using the open structure of the glucokinase (PDB: 1V4T). 


\section{Materials and Methods}

Prediction of the Arg85Trp mutation effect on glucokinase (PDB ID: 1V4T) stability was performed using I-Mutant 3.0 (Capriotti et al., 2005), PoPMuSiC 3.1 [Prediction of Protein Mutant Stability Changes; (Dehouck et al., 2011)], DUET (Pires et al., 2014a) and mCSM [Mutation Cut off Scanning Matrix; (Pires et al., 2014b)] web servers. Otherwise, structural modeling was performed using the Xray crystallographic structure of glucokinase (PDB ID: 1V4T) determined at resolution of $3.4 \AA$ (Kamata et al., 2004). Initially, the water molecules and heteroatoms were removed; polar hydrogens were added. The native structure underwent 10000 steps of steepest-descent energy minimization to remove atomic overlaps or improper geometries, then it was equilibrated for 50000 run in 100 ps using Nano Molecular Dynamics software (NAMD 2.12) (Phillips et al., 2005) with CHARMM 36 force field. After that, the substitution of the arginine at position 85 by a tryptophan residue was introduced into the energy minimized structure using Visual Molecular Dynamics (VMD) (Humphrey et al., 1996) and again energy minimization was carried out.

Analysis and visualization of interactions established between the native Arg85 or the mutated Trp85 and the surrounding amino acids were performed using Accelrys Discovery Studio Visualizer software version 4.1 (http://www.accelrys.com). Lastly, the native and mutated structures were superposed in order to assess likely changes in the three-dimensional (3D) structures.

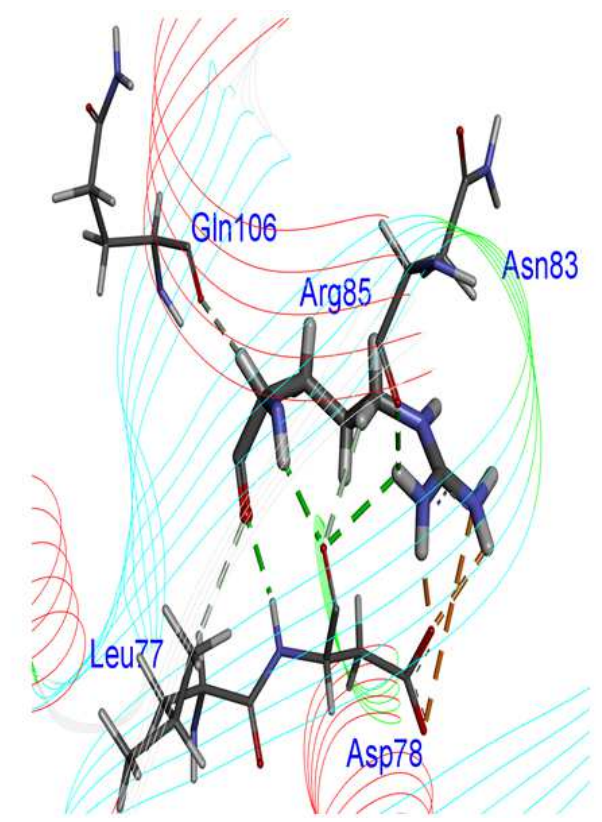

(a)

\section{Results and Discussion}

The stability of the mutated glucokinase was predicted to be altered by all the tested web servers (I-Mutant3.0, PoPMuSiC 3.1, DUET and mCSM) which assess the Arg85Trp mutation to be destabilizing (Table 1).

Structural modeling of the native structure revealed that in the open structure of the glucokinase (PDB: $1 \mathrm{~V} 4 \mathrm{~T}$ ) the arginine 85 is located in a $\beta$ sheet ( $\beta 2$ sheet), near a loop structure (Leu79- Asn83) implicated in the active site of the enzyme (Bouldjennet et al., 2019; Petit et al., 2011).

In the native optimized structure, the residue $\operatorname{Arg} 85$ is involved in different types of interactions with Asp78 residue: One carbon hydrogen bond, three hydrogen bonds (H-bond) and three attractive charge interactions. Furthermore, it establishes two carbon hydrogen bonds with Leu77 and Gln106 and one hydrogen bond with Asn83, (Fig. 1a). These interactions are modified when this small basic arginine residue is substituted by a large hydrophobic tryptophan Trp85. Indeed, in the mutated structure, the new Trp85 residue loses all interactions established between the Arg85 and surrounding residues except three hydrogen bonds with Asp78 residue and the carbon hydrogen bonds with Leu77 and Gln106. Moreover, the three attractive interactions between Arg85 and Asp78 are replaced by pi-pi stacked interaction with His 105 and pi-alkyl interaction with Met87 (Fig. 1b).

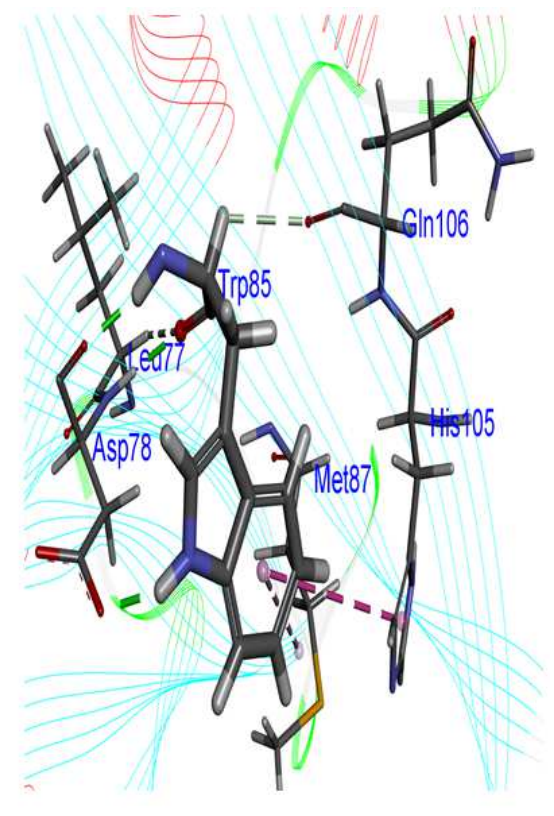

(b)

Fig. 1: View of interactions between; (a) Arg85 in the native structure and surrounding residues; (b) Trp85 in the muted structure and surrounding residues. Dotted lines correspond to interactions: green to hydrogen bonds, light grey to carbon hydrogen bonds, orange to attractive charge interactions, dark and light pink to pi-pi stacked and pi-alkyl interactions, respectively 


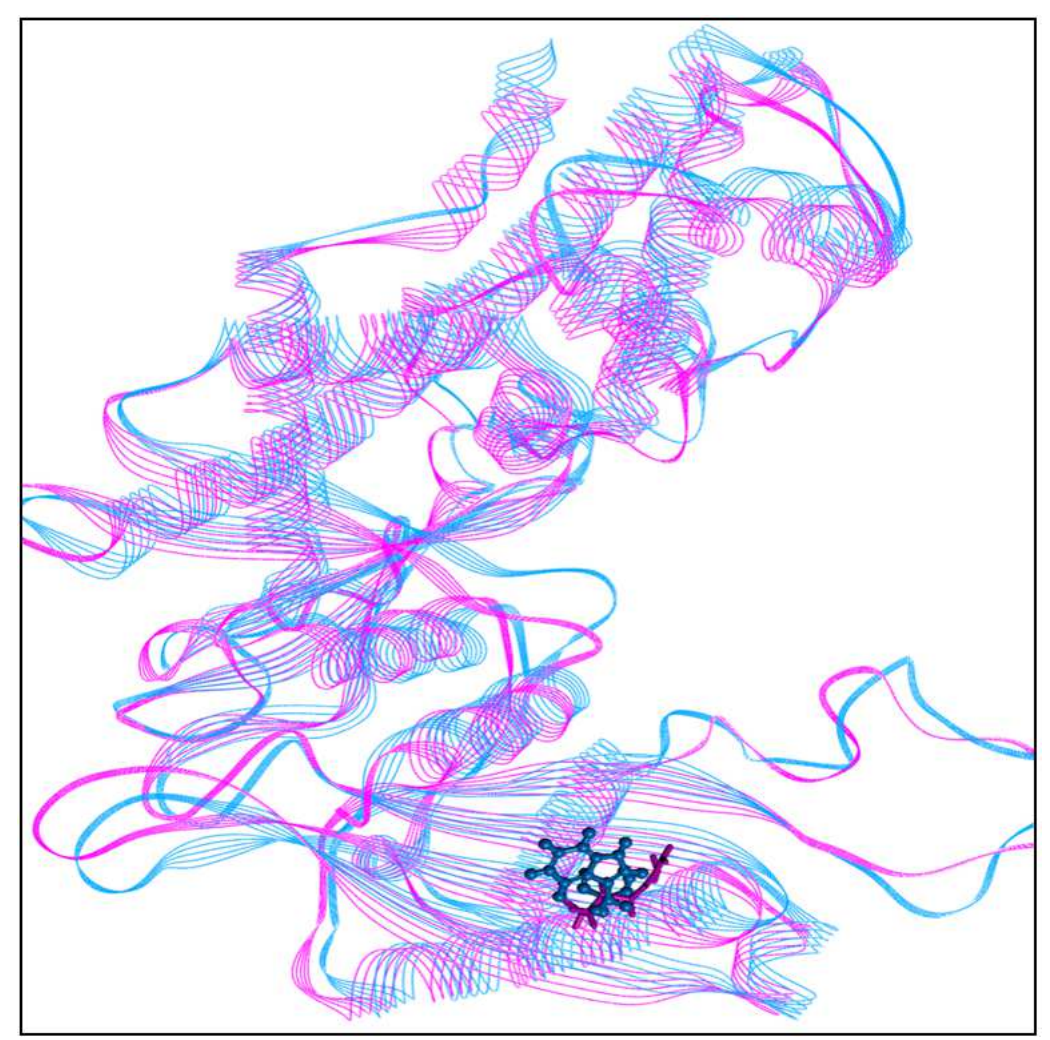

Fig. 2: Superimposition of the native (pink) and mutated glucokinase (bleu). Protein backbones are shown in line ribbon representation

Table 1: In silico predictions of the impact of the Arg85Trp mutation on GCK stability (PDB: 1V4T)

Tools predicting mutation effect

I-Mutant v3.0

PoPMuSiC 3.1

DUET

mCSM

\begin{tabular}{lllll}
\hline Effect on stability & Destabilizing & & -0.585 \\
\hline$\left.\Delta \Delta \mathbf{G}^{*} \mathbf{( K c a l} / \mathbf{m o l}\right)$ & -0.51 & 0.07 & -0.463 & \\
\hline
\end{tabular}

$* \Delta \Delta \mathrm{G}$ : Gibbs free energy.

These interactions changes within the mutated structure are translated by a change in the 3D structure of the mutated protein compared to the native one (Fig. 2).

We should mention that mutations at the Asp78 which loses some interactions with the Trp85 compared to $A r g 85$ and the Asn83 residue which loses interaction with the Trp85, have been linked to MODY phenotype (Massa et al., 2001; Thomson et al., 2003).

\section{Conclusion}

Results of bioinformatic approaches corroborate with the co-segregation of the Arg85Trp glucokinase variant with hyperglycemia. This study supports the deleterious effect of this variation on glucokinase function. However, functional study is needed to highlight altered enzyme's parameters resulting from the detected mutation.

\section{Author's Contributions}

Faiza Bouldjennet: Designed the study and drafted the manuscript.

Souhila Bouaziz-Terrachet: Performed molecular modeling study, reads and approved the manuscript.

Malha Azzouz: Reads and approved the manuscript.

Rachida Raache (Phd Supervisor): Reads and approved the manuscript.

\section{Ethics}

This article is original and contains unpublished material. The corresponding author confirms that all of 
the other authors have read and approved the manuscript and no ethical issues involved.

\section{References}

Barrio, R., C. Bellanne-Chantelot, J.C. Moreno, V. Morel and H. Calle et al., 2002. Nine novel mutations in Maturity-Onset Diabetes of the Young (MODY) candidate genes in 22 Spanish families. J. Clin. Endocrinol. Metab., 87: 2532-2539.

DOI: $10.1210 /$ JCEM.87.6.8530

Bouldjennet, F., A. Hireche, N. Kechout, S. BouazizTerrachet and M. Azzouz et al., 2019. Clinical characteristics of Algerian subjects with MODY p.R85W glucokinase mutation-in silico assessment of p.R85W effect on glucokinase structure and function. Meta Gene, 19: 268-275. DOI: 10.1016/J.MGENE.2018.12.013

Capriotti, E., P. Fariselli, R. Calabrese and R. Casadio, 2005. Predicting protein stability changes from sequences using support vector machines. Bioinformatics, 21: ii54-ii58.

DOI: 10.1093/BIOINFORMATICS/BTI1 109

Capuano, M., C.M. Garcia-Herrero, N. Tinto, C. Carluccio and V. Capobianco et al., 2012. Glucokinase (GCK) mutations and their characterization in MODY2 children of Southern Italy. PLoS One, 7: e38906-e38906.

DOI: 10.1371/JOURNAL.PONE.0038906

Chevre, J.C., E.H. Hani, P. Boutin, M. Vaxillaire and H. Blanche et al., 1998. Mutation screening in 18 caucasian families suggest the existence of other MODY Genes. Diabetologia, 41: 1017-1023. DOI: $10.1007 / \mathrm{S} 001250051025$

Dehouck, Y., J.M. Kwasigroch, D. Gilis and M. Rooman, 2011. Popmusic 2.1: A web server for the estimation of protein stability changes upon mutation and sequence optimality. BMC Bioinform., 12: 151-151. DOI : $10.1186 / 1471-2105-12-151$

Estalella, I., I. Rica, G. Perez de Nanclares, J.R. Bilbao and J.A. Vazquez et al., 2007. Mutations in GCK and Hnf-lalpha explain the majority of cases with clinical diagnosis of MODY in Spain. Clin. Endocrinol. (Oxf), 67: 538-546. DOI: $10.1111 /$ J.1365-2265.2007.02921.X

Humphrey, W., A. Dalke and K. Schulten, 1996. Vmd: Visual molecular dynamics. J. Mol. Graph., 14: 33-38, 27-28.

DOI: 0.1016/0263-7855(96)00018-5

Kamata, K., M. Mitsuya, T. Nishimura, J. Eiki and Y. Nagata, 2004. Structural basis for allosteric regulation of the monomeric allosteric enzyme human glucokinase. Structure, 12: 429-438.

DOI: 10.1016/J.STR.2004.02.005
Massa, O., F. Meschi, A. Cuesta-Munoz, A. Caumo and F. Cerutti et al., 2001. High prevalence of glucokinase mutations in Italian Children with MODY. Influence glucose tolerance, First-phase insulin response, insulin sensitivity and BMI. Diabetologia, 44: 898-905.

DOI: $10.1007 / \mathrm{S} 001250100530$

Petit, P., M. Antoine, G. Ferry, J.A. Boutin and A. Lagarde et al., 2011. The active conformation of human glucokinase is not altered by allosteric activators. Acta Crystallogr D Biol. Crystallogr, 67: 929-935. DOI: 10.1107/S0907444911036729

Phillips, J.C., R. Braun, W. Wang, J. Gumbart and E. Tajkhorshid et al., 2005. Scalable molecular dynamics with NAMD. J. Comput. Chem., 26: 1781-1802. DOI: 10.1002/JCC.20289

Pires, D.E., D.B. Ascher and T.L. Blundell, 2014a. Duet: A server for predicting effects of mutations on protein stability using an integrated computational approach. Nucleic Acids Res., 42: W314-W319. DOI: 10.1093/NAR/GKU411

Pires, D.E., D.B. Ascher and T.L. Blundell. 2014b. mCSM: Predicting the Effects of mutations in proteins using graph-based signatures. Bioinformatics, 30: 335-342.

DOI: 10.1093/BIOINFORMATICS/BTT691

Thomson, K.L., A.L. Gloyn, K. Colclough, M. Batten and L.I. Allen et al., 2003. Identification of 21 Novel Glucokinase (GCK) Mutations in UK and European Caucasians with Maturity-Onset Diabetes of the Young (MODY). Hum Mutat., 22: 417-417. DOI: 10.1002/HUMU.9186

Vionnet, N., M. Stoffel, J. Takeda, K. Yasuda and G.I. Bell et al., 1992. Nonsense mutation in the glucokinase gene causes early-onset non-insulindependent diabetes mellitus. Nature, 356: 721-722. DOI: $10.1038 / 356721 \mathrm{a} 0$ 